\title{
PLANIFICACIÓN ANTICIPADA DE LAS DECISIONES EN EL DISTRITO SANITARIO CÓRDOBA Y GUADALQUIVIR, ESPAÑA
}

\begin{abstract}
José Manuel Jiménez Rodríguez ${ }^{1}$
Resumen: Objetivo: Determinar el conocimiento, actitud y planificación de la voluntad vital anticipada (VVA) en Atención Primaria, e identificar factores asociados. Material/Métodos: Estudio observacional descriptivo transversal. Población diana: médicos y enfermeros adscritos a 15 Unidades de Gestión Clínica del Distrito Sanitario Córdoba-Guadalquivir (España). Muestreo: Multietápico cuya $\mathrm{N}=269$. Instrumento: Cuestionario validado. Análisis estadístico: Descriptivo y bivariante de los datos obtenidos. Resultados: Edad media de los profesionales 51 ańos (Dt. 8,2) siendo mujeres el 60,9\%. El 55,8\% fueron médicos y el 79,3\% propietarios. La puntuación media años de servicio fue 23,5 (Dt. 9,4). El 50,9\% trabajaba en ámbito rural. La puntuación media del conocimiento fue 5,1 (Dt. 2,09). El 98,4\% considera que la VVA está regulada en Andalucía. El 56,9\% no ha leído su documento y el 78,1\% no ha leído la guía de planificación. El 65,6 no ubica el registro de VVA. El $89,3 \%$ no planifica en el último ańo; quien planifica lo hace $<3$ veces. El p-valor de la planificación es: $(\mathrm{p}=0,838)$ respecto del sexo; $(\mathrm{p}=0,521)$ edad; $(\mathrm{p}=0,841)$ ámbito de trabajo, $(\mathrm{p}=0,543)$ categoría profesional; $(\mathrm{p}=0,934)$ años de servicio; $(\mathrm{p}=0,210)$ tipo de contrato. Conclusiones: Pocos profesionales sanitarios planifican con el paciente la VVA, no identificándose factores independientes asociados al estudio.
\end{abstract}

Palabras clave: conocimiento, actitud, planificación anticipada de las decisiones, atención primaria

\section{Advance planning decisions at Cordoba and Gualdaquivir Health District, Spain}

\begin{abstract}
Objectives: Determine the knowledge, attitude and advance care planning in primary care and to identify associated factors. Material /Methods: Type of study: Cross-sectional multicentric study. Study population: Physicians and nurses of 15 Clinical Management Units at Cordoba-Guadalquivir Health District (Spain). Sampling: Multistage with N=269. Tools: Validated questionnaire. Analysis: Descriptive and bivariate statistical analyses were done. Results: Mean age of 51 years professional (SD 8.2) and $60.9 \%$ of them were women. About $55.8 \%$ of participants were physicians and $79.3 \%$ had permanent posts. Mean working years was 23.5 (SD 9.4) and about 50.9\% of participants worked at rural areas. Mean knowledge score was 5.1 (SD 2.09). 98.4\% believe early vital will is regulated in Andalusia. About 56.9\% have not read the regulatory document and $78.1 \%$ have not read the planning guidebook. More than 65\% don't know the corresponding registry office. $89.3 \%$ do not plan in the last year; who planned did that $<3$ times. The $\mathrm{p}$ value of planning is: $(\mathrm{p}=0.838)$ about sex; $(\mathrm{p}=0.521)$ age; $(\mathrm{p}=0.841)$ scope of work, $(\mathrm{p}=0.543)$ professional category; $(\mathrm{p}=0.934)$ years of service; $(\mathrm{p}=0.210)$ type of contract. Conclusions: Few medical practitioners plan with the patient. No independent study identifies factors.
\end{abstract}

Key words: knowledge, attitude, advanced care directives, primary care

\section{Planejamento antecipado das decisóes no distrito de saúde de Córdoba e Guadalquivir, Espanha}

Resumo: Objetivo: determinar o conhecimento, atitude e o planejamento da vontade vital antecipada (VVA) na atenção primária à saúde, e identificar fatores associados. Materiais /métodos: Estudo observacional descritivo transversal. População-alvo: médicos e enfermeiros pertencentes a 15 unidades de Gestáo Clínica de Saúde do Distrito Sanitário Cordoba-Guadalquivir (Espanha). Amostragem: Multistage cujo $\mathrm{N}=269$. Instrumento: Questionário validado. Análise estatística: descritiva e bivariante dos dados obtidos. Resultados: a idade média dos profissionais é de 51 anos (DT. 8.2) sendo 60,9\% a representação de mulheres. 55,8\% foram médicos e 79.3\% proprietários. A pontuaçáo média foi de anos de serviço foi de 23,5 (DT. 9.4). 50,9\% trabalhavam nas zonas rurais. A pontuação média de conhecimento foi de 5.1 (DT. 2.09). 98.4\% considera que a VVA está regulamentada na Andaluzia. 56,9\% não leu seu documento e 78,1\% náo leu o guia de planejamento. Os 65,6\% náo localizam o registro da VVA. 89,3\% não planejam no último ano; quem planeja, o faz $<3$ vezes. O p-valor do planejamento é: $(\mathrm{p}=0$, 838) em relação ao sexo; $(\mathrm{p}=0,521)$ idade; $(\mathrm{p}=0,841)$ âmbito de trabalho, $(\mathrm{p}=0,543)$ categoria profissional; $(\mathrm{p}=0,934)$ anos de serviço; $(p=0,210)$ tipo de contrato. Conclusôes: Poucos profissionais de saúde planejam a VVA com o paciente, náo identificando fatores independentes associados com o estudo.

Palavras-chave: conhecimento, atitude, planejamento antecipado das decisões, atenção primária à saúde

\footnotetext{
${ }^{1}$ Servicio Andaluz de Salud, Distrito Sanitario Córdoba y Guadalquivir, Córdoba, España Correspondencia: jose.jimenez.rodriguez.sspa@juntadeandalucia.es
} 


\section{Introducción}

La Voluntad Vital Anticipa (VVA) es aquella herramienta que permite delimitar y programar las actuaciones clínicas previas a la muerte. Manifestación expresa del consentimiento informado, es el documento (formulario escrito de carácter específico) en el que se plasman los deseos y preferencias de los pacientes ante actuaciones sanitarias al final de la vida, para ser utilizado cuando el paciente se encuentre en situación de incapacidad de hecho $(1,2)$. Dicha VVA tiene sus precedentes en la sentencia del Tribunal de Minnesota (1905) y la sentencia de Nueva York (1914), las cuales reconocen como derecho inherente al ser humano poder decidir respecto de qué hacer con el propio cuerpo en los momentos finales de la vida; posteriormente, esta quedará refrendada en la sentencia dictada por el Tribunal Supremo de California (1957) con el caso "Salgo", la sentencia del Tribunal Supremo de New Jersey (1976) con el caso de "Quinlan" y la sentencia dictaminada por el Tribunal Supremo de los Estados Unidos (1990) con el caso de "Cruzan" $(3,4)$. Con la entrada en vigor de la ley 41/2002, básica reguladora de la autonomía del paciente y de derechos y obligaciones en materia de información y documentación clínica, España comienza a regular el derecho a la autodeterminación del paciente en aquellas cuestiones relacionadas con sus deseos y decisiones sanitarias (respecto de las actuaciones clínicas que le puedan ser aplicadas), entre las que se encuentran los cuidados paliativos $(5,6)$. El derecho a una muerte programada y enaltecida, lejos de prácticas iatrogénicas basadas en el encarnizamiento terapéutico, hacen de la VVA una alternativa progresista a los principios ortodoxos de la ética médica tradicional $(7,8)$. En la Comunidad Autónoma de Andalucía, y por ende en la ley 5/2003, de declaración de voluntad vital anticipada, dicha prácticas comienzan a ser regulada e integradas en la cartera de servicios del Sistema Sanitario Público, como una prestación más a la que el paciente puede optar de forma deliberada(9-11). Desde este momento, la VVA queda al amparo de los poderes públicos, la administración sanitaria, el cuerpo facultativo y el propio paciente, ello desde un paradigma basado en el modelo bioético. La VVA y el proceso de muerte pasan a formar parte de una decisión personal y madurada, transferible en aquellos casos en que la capacidad de decidir merma hasta el extremo $(12,13)$. Los profesionales sanitarios de Atención Primaria (AP) juegan un papel de suma importancia en el desarrollo de dicha VVA y el documento que la desarrolla, el cual puede verse irrumpido por condicionamientos relativos al conocimiento, la actitud e implicación que estos puedan tener respecto del mismo(14-17). Más de un decenio en convivencia con dicha VVA, nos invita a reflexionar acerca de los posicionamientos que puedan tener los profesionales de la salud respecto de una práctica sanitaria extendida en todo el territorio español, así como de aquellos otros elementos emergentes y adscritos al propio sistema sanitario que puedan estar influyendo en una garante planificación de la VVA(18). Cumpliendo con nuestro objeto de estudio, se hace necesario un exhaustivo análisis sobre el grado de conocimiento, actitud y nivel de planificación de la VVA de mano de estos profesionales, como profundizar sobre aquellas otras variables que, sirviendo como indicador comparativo, pueden establecer diferencias en el desarrollo de dicha práctica desde $\mathrm{AP}(19,20)$.

\section{Material y métodos}

Estudio observacional descriptivo transversal de tipo multicéntrico. La población de estudio queda acotada al cuerpo de profesionales sanitarios: médicos/as y enfermeros/as de AP adscritos al Distrito Sanitario Córdoba y Guadalquivir (Córdoba, España). Se tomó como referencia 15 de las 20 Unidades de Gestión Clínica (UGC) adheridas a dicho Distrito Sanitario de AP. Se realizó un muestreo de tipo multietápico (estratificado y sistemático) en cuanto al ámbito de actuación y categoría profesional se refiere, cuya $\mathrm{N}$ fue igual a 269 individuos. Se consideró como criterio de inclusión encontrarse activo y con más de un año de servicio en el Sistema Sanitario de Andalucía durante el periodo de recogida de datos (2014). Se administró un cuestionario previamente validado, que dio respuesta a los objetivos del estudio (ver anexo 1)(21). Las variables independientes fueron el sexo, la edad, la UGC de pertenencia, la categoría profesional, el tipo de contrato y los años de servicio. Las tres variables dependientes 
(conocimiento, actitud y nivel de planificación) se midieron mediante los 17 ítems que conforman el cuestionario con escala numérica (0-10), así como, con respuestas dicotómicas (si/no, ns/nc). Para la interpretación de los resultados se hizo un análisis sociodescriptivo, mediante distribución de frecuencias, porcentaje, máximos y mínimos, media y desviación típica, así como un análisis bivariante de los datos obtenidos con su IC del 95\%. Para asumir los supuestos de normalidad se utilizaron las pruebas estadísticas T-student y Mann-Whitney, así como para las variables independientes con tres o más respuestas se utilizó la prueba estadística ANOVA. Para el análisis de los datos obtenidos de utilizó el programa informático $\mathrm{R}$ para Windows, versión $3.02^{\circledR}$.

\section{Resultados}

Se obtuvo 269 cuestionarios cumplimentados de los 388 cuestionarios entregados. La media de edad de estos profesionales fue de 51 años (rango 25-63; Dt. 8,22). El 60,9\% de los participantes fueron mujeres. La categoría profesional predominante fue la de médico con un 55,8\%. El 79,3\% era propietario de la plaza y el 50,9\% trabajaba en ámbito rural. La media de años de servicio fue de 23,5 (Dt. 9,47). La puntuación media del conocimiento fue de 5,1 (Dt. 2,09). El $98,4 \%$ de estos profesionales considera que la VVA está regulada en Andalucía. El 56,9\% no había leído el documento y el 78,1\% no había leído la guía de apoyo a los profesionales para la planificación anticipada. El 65,6\% no ubicó el registro provincial de las VVA (ver tabla 1). El 7,8 (Dt. 2,17) de los profesionales vio conveniente que los pacientes planifiquen sus deseos sanitarios dejándolos por escrito y el 7,8 (Dt. 2,29) consideró la declaración de VVA un instrumento útil para los profesionales sanitarios a la hora de tomar decisiones sobre un paciente. El 9,1 (Dt. 1,52) respetaría los deseos expresados por un paciente en una declaración de VVA y el 8,4 (Dt. 2,11) cree que, si el paciente nombrase un representante en la VVA, facilitaría la toma de decisiones a los profesionales sanitarios. Aunque el 7,9\% (Dt. 2,35) recomendaría la planificación a sus pacientes, tan solo un 3,3 (Dt. 3,51) de estos profesionales realizaría su propia VVA en el último año. Por su parte, el 89,3\% no planificó en el último año y quien planificó lo hace menos de 3 veces. El 4,8 (Dt. 3,31) estimó la religión (creencias, valores, etc.) un obstáculo al que el paciente debe de hacer frente a la hora de llevar a cabo su propia declaración de VVA, y el 2,8 (Dt. 3,67) consideró que sus propias creencias religiosas podrían entran en conflicto con su práctica profesional a la hora de planificar la VVA con sus pacientes. El 3,3 (Dt. 3,34) de los profesionales entendió que el deseo expresado por los pacientes en la VVA podría entra en conflicto con lo dictado en el Código Deontológico. Respecto de la emisión de información de la VVA como prestación, el 3,9\% (Dt. 3,43) pensó que en su UGC o Centro de Salud la información sobre la VVA corresponde a los profesionales no sanitarios (trabajadores/as sociales y/o personal administrativo). Finalmente, en relación al análisis entre la planificación y las variables independientes no se encuentra diferencias significativas. En la distribución de respuesta su puede comprobar como para el sexo la $\mathrm{p}=0,838(X 2=0,069)$; para la edad la $\mathrm{p}=0,521(X 2=1,474)$; para el ámbito de trabajo la $\mathrm{p}=0,841 \quad(X 2=0,061)$; para la categoría profesional la $\mathrm{p}=0,543(X 2=0,484)$; para los años de servicio la $\mathrm{p}=0,934(X 2=0,196)$; y para el tipo de contrato la $\mathrm{p}=0,210(X 2=3,310)($ ver tabla 2$)$.

\section{Discusión}

La VVA y las cuestiones de muerte son prioridad y controversia de las políticas sanitarias. Ofertada en cartera de servicios del Servicio Andaluz de Salud, se convierte en una prestación al alcance de cualquier ciudadano que quiera solicitarla. Considerada la $\mathrm{AP}$ el escenario más adecuado para iniciar dicha prestación, a través de este se puede establecer un verdadero clima de empatía y confianza entre el profesional y el paciente, condición necesaria para el garante derecho a la planificación de la VVA $(22,23)$. Ello lleva implícito un conocimiento mínimo sobre esta herramienta y su desarrollo, así como una actitud positiva y abierta a prácticas sanitarias alternativas. Los sujetos investigados en nuestro estudio dan una puntuación media del conocimiento respecto de la VVA de 5,1 (Dt. 2,09), dato que difiere poco de los resultados extraídos de otros estudios de semejante calado(24). Pese a que el 7,8\% (media) de estos profesionales considera conveniente que los pacientes planifiquen sus deseos sanitarios y que el 7,9 (media) recomendaría la planificación a sus pacientes, tan solo el 
Planificación anticipada de las decisiones - José Manuel Jiménez Rodríguez

Tabla 1. Análisis del conocimiento de la VVA según variables independientes

\begin{tabular}{|c|c|c|c|c|c|c|c|c|}
\hline \multicolumn{6}{|c|}{ ¿Qué puntuación daría a sus conocimientos sobre las VVA? } & \multicolumn{2}{|c|}{$\begin{array}{l}\text { I.C. para media al } \\
95 \%\end{array}$} & \multirow[t]{2}{*}{ Prueba Estadística } \\
\hline & & $\mathrm{N}$ & Media & Desv. típ & Err. típ & L.I. & L.S. & \\
\hline \multirow[t]{2}{*}{ Sexo } & Varón & 105 & 5,22 & 2,09 & 0,20 & 4,81 & 5,62 & \multirow{2}{*}{$\begin{array}{l}\mathrm{T}=0,298 ; \\
\text { g.l. }=266 ; p=0,766\end{array}$} \\
\hline & Mujer & 163 & 5,14 & 2,09 & 0,16 & 4,82 & 5,46 & \\
\hline \multirow[t]{3}{*}{ Edad } & Menores 45 & 56 & 5,18 & 1,88 & 0,25 & 4,68 & 5,68 & \multirow{3}{*}{$\begin{array}{l}\mathrm{F}=0,012 ; \mathrm{g} .1 .=2 ; \\
\text { g.l. }=247 ; \mathrm{p}=0,988\end{array}$} \\
\hline & $\begin{array}{l}\text { Entre } 46 \\
\text { y } 55\end{array}$ & 109 & 5,21 & 2,22 & 0,21 & 4,79 & 5,63 & \\
\hline & Mayores 56 & 85 & 5,16 & 2,16 & 0,23 & 4,70 & 5,63 & \\
\hline \multirow[t]{2}{*}{ Ámbito Rural } & Rural & 136 & 5,38 & 1,96 & 0,17 & 5,04 & 5,71 & \multirow{2}{*}{$\begin{array}{l}\mathrm{T}=1,623 ; \\
\text { g.l. }=266 ; p=0,106\end{array}$} \\
\hline & Urbano & 132 & 4,96 & 2,20 & 0,19 & 4,58 & 5,34 & \\
\hline \multirow[t]{2}{*}{ Categ. Profesional } & Médico/a & 150 & 4,95 & 2,12 & 0,17 & 4,61 & 5,29 & \multirow{2}{*}{$\begin{array}{l}T=-1,940 ; \\
\text { g.l. }=266 ; p=0,053\end{array}$} \\
\hline & Enfermero/a & 118 & 5,45 & 2,03 & 0,19 & 5,08 & 5,82 & \\
\hline \multirow[t]{3}{*}{ Tipo Contrato } & Propietario/a & 210 & 5,14 & 2,16 & 0,15 & 4,84 & 5,43 & \multirow{3}{*}{$\begin{array}{l}F=0,416 ; g .1 .=2 ; \\
\text { g.l. }=262 ; p=0,660\end{array}$} \\
\hline & Interino/a & 23 & 5,52 & 1,50 & 0,31 & 4,87 & 6,17 & \\
\hline & Eventual & 32 & 5,03 & 1,96 & 0,35 & 4,32 & 5,74 & \\
\hline \multirow[t]{3}{*}{ Años Trabajados } & - de 19 años & 79 & 5,15 & 1,99 & 0,22 & 4,71 & 5,60 & \multirow{3}{*}{$\begin{array}{l}F=0,096 ; \text { g.l. }=2 ; \\
\text { g.l. }=253 ; p=0,909\end{array}$} \\
\hline & $\begin{array}{l}\text { Entre } 20 \\
\text { у } 29\end{array}$ & 102 & 5,28 & 2,28 & 0,23 & 4,84 & 5,73 & \\
\hline & + de 30 años & 75 & 5,19 & 2,04 & 0,24 & 4,72 & 5,66 & \\
\hline
\end{tabular}

Tabla 2. La Planificación de la Voluntad Vital Anticipada desde el conocimiento: análisis de variables

\begin{tabular}{|c|c|c|c|c|c|c|c|c|c|c|c|c|c|c|}
\hline \multicolumn{15}{|c|}{ Planifica con sus pacientes (previa y en consulta) la Voluntad Vital Anticipada } \\
\hline \multicolumn{2}{|l|}{$\begin{array}{l}\text { Sexo } \\
(\%)\end{array}$} & \multicolumn{3}{|c|}{$\begin{array}{l}\text { Edad } \\
(\%) \\
\end{array}$} & \multicolumn{2}{|c|}{$\begin{array}{l}\text { Ámbito } \\
(\%)\end{array}$} & \multicolumn{2}{|c|}{$\begin{array}{l}\text { Categoría Pro- } \\
\text { fesión }(\%)\end{array}$} & \multicolumn{3}{|c|}{$\begin{array}{l}\text { Tipo Contrato } \\
(\%)\end{array}$} & \multicolumn{3}{|c|}{$\begin{array}{l}\text { Años Servicio } \\
(\%)\end{array}$} \\
\hline $\mathrm{H}$ & M & -45 & $46-55$ & +56 & Rural & $\begin{array}{l}\text { Urba- } \\
\text { no }\end{array}$ & MAP & ATS & Pro. & Int. & Eve. & -19 & $20-29$ & +30 \\
\hline $\mathrm{No}=$ & $\mathrm{No}=$ & $\mathrm{No}=$ & $\mathrm{No}=$ & $\mathrm{No}=$ & $\mathrm{No}=$ & $\mathrm{No}=$ & $\mathrm{No}=$ & $\mathrm{No}=$ & $\mathrm{No}=$ & $\mathrm{No}=$ & $\mathrm{No}=$ & $\mathrm{No}=$ & $\mathrm{No}=$ & $\mathrm{No}=$ \\
\hline 90 & 88,9 & 90,2 & 85,9 & 91,3 & 89,8 & 88,9 & 88,2 & 90,9 & 89 & 100 & 85,1 & 87,6 & 88,8 & 90 \\
\hline \multicolumn{2}{|c|}{$\begin{array}{l}\chi^{2}=0,069 ; \\
\text { g.l.=1; } \\
p=0,838\end{array}$} & \multicolumn{3}{|c|}{$\begin{array}{l}\chi^{2}=1,474 ; \text { g.l. }=2 \\
\mathrm{p}=0,521\end{array}$} & \multicolumn{2}{|c|}{$\begin{array}{l}\chi^{2}=0,061 ; \\
\text { g.l. }=1 ; \\
p=0,841\end{array}$} & \multicolumn{2}{|c|}{$\begin{array}{l}\chi^{2}=0,484 ; \\
\text { g.l. }=1 ; \\
p=0,543\end{array}$} & \multicolumn{3}{|c|}{$\begin{array}{l}\chi^{2}=3,310 ; \text { g.l. }=2 \\
\mathrm{p}=0,210\end{array}$} & \multicolumn{3}{|c|}{$\begin{array}{l}\chi^{2}=0,196 ; \text { g.l. }=2 \\
\mathrm{p}=0,934\end{array}$} \\
\hline
\end{tabular}

$10,7 \%$ de estos profesionales planifica la VVA previamente y en consulta durante el último año. Únicamente, el 21,9\% ha leído la guía de apoyo a los profesionales para la planificación anticipada (publicada en Andalucía en el año 2013), lo que nos invita a pensar en la falta de habilidad para poder encarar en tema como uno de los motivos del bajo número de planificaciones anticipadas desde AP. Ello, unido al desconocimiento de los puntos de registro de la VVA en más de la mitad de los casos, que impide derivar al paciente y suplir carencias respecto del conocimiento del documento y la guía que desarrolla la planificación, nos hace poner el acento sobre la formación, motivación e implicación de los profesionales de la salud. Un conocimiento y una actitud positiva, así como la ausencia de discrepancias entre las variables de segmentación, nos hace pensar en componentes de tipo organizativo (tiempo de respuesta, demora en las consultas), síndrome de burnout, etc., como elementos responsables de la planificación realizada en 2014. Una mayor información y formación, y un aumento en el nivel de concienciación de los profesionales respecto de la VVA, garantizaría la expansión en la planificación de la VVA(25). Desde el sistema sanitario se hace necesaria una 
intervención, basada en directrices formativas y de sensibilización, que avale el impulso y éxito de una prestación implantada desde hace más de un decenio, y que a su vez vislumbre de una forma más específica el modo de registrar y participar en la planificación de la VVA de los ciudadanos interesados(26).

\section{Responsabilidades éticas}

Protección de personas y animales. El autor declara que en dicha investigación no se ha realizado experimento alguno con seres humanos ni con animales.

Confidencialidad de los datos. El autor declara que en este artículo no aparecen datos de pacientes que puedan ser comprometidos y violen la legislación vigente.

Derecho a la privacidad y consentimiento informado. El autor declara que en este artículo no aparecen datos de pacientes.

Conflictos de interés: ninguno.

\section{Agradecimientos}

A la gerencia del Distrito Sanitario Córdoba y Guadalquivir de la provincia de Córdoba y la dirección médica de las diferentes UGC adscritas al mismo, la oportunidad brindada para la puesta en marcha y desarrollo de la investigación planteada. Del mismo modo, la colaboración de todos los profesionales sanitarios y no sanitarios adheridos a dichas UGC, por su participación y tiempo desinteresado en el estudio.

\section{Referencias}

1. Angora F. Voluntades anticipadas versus instrucciones previas en atención primaria. Salud 2000. Política Sanitaria 2008; 116: $14-8$

2. Antolín A, Sánchez M, Miró O. Temporal trend in understanding of and attitudes to advance directives in patient with chronic diseases. Gac Sanit 2011; 25(5): 412-418.

3. Requero JL. El testamento vital y las voluntades anticipadas: aproximación al ordenamiento español. Ciclo de ética y humanidades médicas. ICOMZ. Zaragoza: Instituto de Bioética y Ciencias de la Salud; 2002.

4. Seoane JA. Derecho y planificación anticipada de la atención: panorama jurídico de las instrucciones previas en España. Derecho y Salud España 2006; 14(2): 285-295.

5. Antolín A, et al. Documentos de voluntades anticipadas: mucho camino por recorrer para alcanzar una autonomía plena del paciente. Med Clin Barcelona 2011; Doi:10.1016/j.medcli.2010.04.023.

6. Ley 41/2002 de 14 de noviembre, básica reguladora de la autonomía del paciente y de derechos y obligaciones en materia de información y documentación clínica. BOE 15 de noviembre de 2002; 274: 40126-40132.

7. Casado M. Nuevos materiales de bioética y derecho. México: Fontamara; 2007.

8. Casado M, et al. Sobre las voluntades anticipadas: aspectos bioéticos, jurídicos y sociales. Rev Esp Med Legal. http:// dx.doi.org/10.1016/j.reml.2012.05.006.

9. Ley 5/2003, de 9 de octubre, de declaración de voluntad vital anticipada. BOE 21 de noviembre de 2002; $279: 41231$ 41234.

10. Decreto 59/2012, de 13 de marzo, por el que se regula la organización y funcionamiento del Registro de Voluntades Vitales Anticipadas de Andalucía. BOJA 26 de marzo de 2012; 59: 40-52.

11. Angora F. Voluntades anticipadas versus instrucciones previas en atención primaria. Salud 2000. Pol San 2008; 116: 14-18.

12. Junta de Andalucía. Guía para hacer la voluntad vital anticipada. [Consultado 21 Jun 2015]. Disponible en: http://www. juntadeandalucia.es/salud/sites/csalud/contenidos/Informacion_General/c_2_c_8_voluntad_vital_anticipada/guia. 
Planificación anticipada de las decisiones - José Manuel Jiménez Rodríguez

13. Nebot C, Ortega B, Mira JJ, Ortiz L. Morir con dignidad. Estudio sobre voluntades anticipadas. Gac Sanit 2010; 24(6): 437-445.

14. Ley $2 / 2010$, de 8 de abril, de derechos y garantías de la dignidad de la persona en el proceso de la muerte. BOE 25 de mayo de 2010; 127: 45646-45662.

15. Junta de Andalucía. Planificación anticipada de las decisiones. Guía de apoyo para profesionales. [Consultado 21 Jun 2015 ]. Disponible en: http://www.juntadeandalucia.es/salud/sites/csalud/contenidos/Informacion_General/p_3_p_20_planificacion_anticipada_decisiones/planificacion_anticipada_decisiones?=org

16. Jiménez JM, Huertas F, Gómez A, Fajardo C, Valverde FJ. Diferencias en la percepción de conocimientos y actitudes de profesionales sanitarios sobre el documento de voluntades anticipadas. Med Fam And. Atención Primaria 2013; $45: 233$.

17. Arauzo V, Trenado J, Busqueta G y Quintana S. Grado de conocimiento sobre la ley de voluntades anticipadas entre los familiares de los pacientes ingresados en un servicio de medicina intensiva. Med Clin Barc 2010; 134(10): 448-451.

18. Fajardo C, Valverde FJ, Jiménez JM, Gómez A, Huertas F. Grado de conocimiento y actitudes de los profesionales ante el documento de voluntades anticipadas: diferencias entre distintos profesionales y provincias de una misma autonomía. Semergem 2015; 41(3): 139-148.

19. Valverde FJ, Jiménez JM, González V, Huertas F, Gómez A, Fajardo C. Conocimientos y actitudes de los profesionales sanitarios sobre los documento de voluntades anticipadas. Med Fam And 2012; 13(1): 84.

20. Leal M, Rivas JA, Martínez F, Lozano M. Papel del médico de familia en el cumplimiento y registro del documento de instrucciones previas. Semergen 2015; 41(3): 164-167.

21. Jiménez JM. El cuestionario "conocimiento, actitud y planificación ante la voluntad vital anticipada": una perspectiva metodológica desde trabajo social sanitario. Trab Soc y Salud Zarag. 2015; 80(2): 24-34.

22. Santos de Unamuno C. Documentos de voluntades anticipadas: actitud de los pacientes de atención primaria. Atención Primaria 2003; 32: 1-8.

23. Álvarez S, Argüelles L, Álvarez M, Fernández T, Blanco ML, Castillo F. Conocimiento y actitudes de las enfermeras de Atención Primaria sobre las Voluntades Anticipadas. Enfermería Comunitaria RqR 2015; 3(1): 66-77.

24. Fajardo C, Valverde FJ, Jiménez JM, Gómez A, Huertas F. Conocimientos y actitudes de los profesionales sanitarios sobre el documento de voluntades anticipadas. SAMFyC Córdoba 2011; 12(2): 259.

25. Altisent R. Planificación anticipada de la asistencia. Se necesita una revolución educativa. Aten Primaria 2013; 45(8): 402-403.

26. Atienza E, Armaza EJ, Beriain IM. Aspectos bioéticos-jurídicos de las instrucciones previas o testamento vital en el contexto normativo español. Acta Bioethica 2015; 21(2): 163-172.

Recibido: 26 de noviembre de 2015

Aceptado: 14 de febrero de 2016 